\title{
GPS data Mining at Signalized Intersections for Congestion Charging
}

\author{
Wang $\mathrm{Yu}^{1,2} \cdot$ Zhang Dongbo ${ }^{3}$ (D) Zhang $\mathrm{Yu}^{4}$
}

Accepted: 12 January 2022 / Published online: 8 February 2022

(c) The Author(s), under exclusive licence to Springer Science+Business Media, LLC, part of Springer Nature 2022

\begin{abstract}
Nowadays more private car trips have caused worse congestion due to the Covid-19 pandemic in many cities. Congestion charging is one of the taxes that is levied on vehicle owners to reduce urban traffic congestion. One of the most important reasons congestion charging is not accepted by the public is the high cost. Monitoring the state of traffic congestion in real time requires a lot of expensive installations. The purpose of this paper is to make congestion charging more accurate and acceptable using artificial intelligent algorithm. Massive real-time Global Positioning System (GPS) data provides new data for road congestion charging. The queuing length at intersections is an important measurement for the degree of traffic congestion, and it is also the basis for road congestion pricing. GPS positioning cannot provide sufficient position accuracy for lane identification of vehicles. In this study, a comprehensive model consisting of a real-time lane identification model and a real-time queue length estimation model is developed based on the traffic shockwave theory using GPS data. The comprehensive model can identify the lane where the queuing vehicle is located and estimate the real-time queue length of the lane. The proposed models were evaluated using field-collected data in Guangzhou, China. The testing results show that the proposed comprehensive model can identify lanes and estimate queue lengths with satisfactory accuracy. The model proposed in this paper provides real-time data for road dynamic pricing in a cost-effective way, which can promote the implementation of congestion charging in cities.
\end{abstract}

Keywords Congestion charging · Lane identification · Queue length estimation · Traffic shockwave

Extended author information available on the last page of the article 


\section{Introduction}

The ever-increasing urban population and vehicular traffic have made urban traffic problems increasingly prominent. At present, cities suffer from severe traffic congestion problems. Traffic jams have caused time waste, environmental pollution, and increase of accidents. This situation is even more serious in densely populated cities, which bear huge economic losses because of congestion every year.

Current methods to solve traffic congestion mainly include: expanding urban roads, improving public transport, developing Intelligent Transportation System and congestion charging.

(1) Expanding urban roads.

Many facts have shown the increase in road area cannot reduce traffic congestion, and will make traffic congestion more severe. The "Downs' Law" (Downs, 1962) in traffic economics believes that new road will induce new traffic demand, and traffic demand always exceed traffic supply. In the long term, adding roads will make the situation even worse and empirical study in many cities confirmed this argument.

(2) Improving public transport.

Developing public transport includes subsidies for public transport, which can make public transport more attractive, thereby reducing traffic congestion. Nowadays the Covid-19 pandemic has limited the load factor of public transport in many cities. This shortage of public transport will cause worse traffic congestion (Ortego et al., 2020).

(3) Developing Intelligent Transportation System.

ITS (Intelligent Transportation System) is an accurate and efficient comprehensive transportation system, which integrates various advanced technologies. ITS provides urban administrations with an effective way to solve traffic congestion. In addition, ITS provides technology and infrastructures for congestion charging.

(4) Congestion charging.

Congestion charging is a hot issue studied by many scholars in recent years. It refers to the use of price principles in economics to restrict traffic demand, thereby changing traveler behavior and reducing traffic congestion. Pigou (1920) first proposed the congestion charging theory, which pointed out that traffic congestion makes the marginal social cost of road users higher than the marginal private cost, so congestion produces negative externalities. Congestion charging internalizes the congestion costs for cities. Congestion charging can redistribute traffic flow in time and space, so it can improve the efficiency of the entire road network.

Congestion charging can also provide a new source of funding for improving public transport services. Investments in the public transport system permanently require fresh resources. Congestion taxes are significant resources for cities and can stimulate economic growth. Congestion tax not only affects traffic, but also is closely related to financial affairs.

Congestion charging schemes can be divided into two categories according to the scope of implementation: road congestion charging and zone congestion charging. Road congestion charging refers to charging for High Occupancy Toll (HOT) lanes or roads in specific sections and periods. Zone congestion charging refers to charging for zones with frequent large-scale congestion in specific sections and periods. 
The Congestion pricing theory of bottleneck road (Vickrey, 1969) believes that the optimal charge is time-varying, and the charging price is determined by the queue length at the bottleneck intersection. The congestion cost is equal to the externalities generated by road users at the specific time and place. Therefore, congestion cost varies depending on the traffic state. Ideally, the charge should be set dynamically (Bocarejo, 2020). Reasonable congestion charging should be based on traffic state, and differential charge should be established for different zones and times of the day. Dynamic pricing will make the costs for investment and operation of the congestion pricing system very large. The congestion pricing system requires a high number of expensive installations to measure traffic volumes and congestion.

Although congestion charging has captured considerable attention in practice and academia, congestion charging is generally not accepted by the public in practice, and few cities have managed to implement congestion charging (Mehdizadeh \& Shariat-Mohaymany, 2020). There are two main reasons why congestion charging is rejected by public opinion. First, Congestion charging is considered as an additional tax. Second, the cost to build and operate a congestion charging system is too high (Buckingham et al., 2010).

In fact, the principle of congestion charging is fair. It internalizes the external costs of congestion. Congestion charging is conducive to the sustainability and efficiency of urban traffic, which is better than existing fees and taxes (Cipriani et al., 2019).

The cost of the congestion pricing system mainly comes from some expensive installations, most of which are used to measure traffic congestion and identify traffic state. With the widespread use of smart phones, car navigators and other devices, more and more mobile smart devices can be used as mobile traffic sensors to provide real-time GPS data of vehicles. Nowadays, massive trajectory data covers all parts of the urban traffic network, providing rich data for congestion measurement (Wang et al., 2020a, b). If AI technology is used to confirm the state of traffic flow based on massive GPS data, the cost of the congestion pricing system will be greatly reduced.

The queue length of an intersection can reflects the congestion status of the intersection in time. The queuing length at the bottleneck intersection is the basis for road congestion pricing, and it is also an important indicator to identify the delay time, space range and the degree of congestion in urban traffic jams. It's necessary to study how to accurately estimate intersection queue length using GPS data.

At present, lane identification is a difficult subject in the research of queue length at intersections using GPS data. In urban areas, due to the urban canyon effect (multipath interference caused by reflections from high-rise buildings) ( $\mathrm{Li} \& \mathrm{Fu}, 2020)$, the error of commercial GPS modules readings is as high as tens of meters. Such accuracy cannot meet the requirements for determining the vehicles' lane. The lanebased real-time queue length plays a vital role in congestion charging.

Therefore, the question raised by this research is how to use GPS data to identify the lanes of vehicles at signalized intersections in real time, and how to estimate the queue lengths on the individual lanes.

The rest of this paper is organized as follows. The related studies are discussed in Sect. 2. Sect. 3 proposes the research model and framework, including the lane identification model and the real-time queue length estimation model. The test results 
and analysis are described in Sect. 4. The conclusions and future work are presented in Sect. 5.

\section{Literature Review}

\subsection{Related Studies}

Over the years, a large number of researchers have studied the estimation of queue length at intersections. In recent years, real-time queue length estimation using finegrained data has become a new hot research. The estimation methods of queue length can be divided into three categories: (1) the input-output model, (2) the hybrid model, and (3) the traffic shockwave model.

The input-output model (An et al., 2017) derives cumulative arrivals and departures from the fixed-location sensors and then estimates the queue length. This model cannot obtain the spatial distribution of queue length in time, and is not suitable for long queues. The hybrid model (Cai et al., 2014) is based on the input-output model, which requires additional stop bar detector to count the vehicles crossing the stop bar. In addition, the estimation method based on video (Jiang et al., 2019) uses image recognition technique to estimate the length of the queue. This method needs a video detector at the intersection, and the computation cost of the algorithm is high, so the real-time ability is poor. The model based on traffic shockwave theory can describe the complex queuing process in both temporal and spatial dimensions, so it has attracted the attention of many researchers (Gao et al., 2019).

Many real-time queue length estimation methods use fixed-location sensor data, and some others methods (Cai et al., 2014) combine GPS data with fixed-location sensor data. The coverage of these fixed-location sensors is limited, and detectors such as inductive loop sensors are easily damaged in practice, which limits the application of many estimation methods. (Gao et al., 2019) proposed a queue length sensing model based on V2X technology. However, the current transport facilities cannot meet the requirements of the V2X technology.

Nowadays, a large amount of real-time GPS data provides a new resource for the estimation of queue length at intersections. Tan et al., (2021) proposed an approach for queue length estimation through maximum likelihood estimation using vehicle trajectory data. But the quality of historical trajectory data has a great influence on the accuracy of the algorithm. Wang et al., $(2020 \mathrm{a}, \mathrm{b})$ proposed an integer programming model to estimate the queue profile. The approach requires low GPS location error, and is not suitable for real-time queue length estimation in large-scale networks. By identifying the critical points from raw trajectories, Cheng et al., (2011) proposed a queue length estimation method based on shock waves. The estimation method also needs accurate location data. Further, the studies above (Tan et al., 2021; Wang et al., 2020a, b; Cheng et al., 2011) do not address identifying the lane of the queue.

In the application of real-time GPS data, most researchers use vehicle position data to estimate the queue length. Because the intersection is the intersection of multiple roads, the accuracy of map matching is not high when the vehicle approaches the intersection. Moreover, the accuracy of GPS orientation data is also low due to 
the deceleration, stop, and steering of the vehicle. Therefore, near the intersection, there is often a large error in the GPS position data of vehicles.

Moreover, compared with fixed detector data, mobile sensor data has the biggest flaw in the uncertainty. The number of vehicles that can provide GPS data is uncertain, which is the biggest challenge in using GPS data. Location error and high uncertainty make real-time queue analysis need to solve many problems using GPS data at intersections.

In the research on lane identification, most subjects are studying how to use machine vision technology to confirm lanes for the intelligent vehicle navigation and autonomous vehicles (Wang et al., 2019; Zhang et al., 2020). (Madhu et al., 2020) proposed a methodology of extracting vehicle trajectory data to analyze the following behavior of vehicles such as changing lanes. These studies are not applicable to the lane identification for queue length estimation at intersections.

Rompis et al. (Semuel et al., 2018) proposed a method for identifying the lane of the GPS instrumented vehicles queuing at signalized intersections. Based on the time when the probe vehicle joined the queue and the distance between the probe vehicle and the stop bar, the queuing shockwave speed was calculated in their method. In actual traffic, there are large errors in the GPS location data of vehicles at intersections, which will seriously affect the accuracy of the parameters. In addition, the method is only suitable for undersaturated traffic flow conditions. In over-saturation, the queue lengths of lanes with different turns may also be different, which is the same as the case of undersaturation. Under the condition of over-saturation, lanebased queue length estimation is an important content to be studied.

The lane-based real-time queue length is an important basis for road pricing. As mentioned above, due to GPS location errors, the lane identification problem of queuing vehicles has not been resolved. The research on real-time queue length estimation for signalized intersections using GPS data is still incomplete.

This paper studies how to use GPS data to determine the lane of queuing vehicles, and estimates the real-time queue length at signalized intersections. This paper obtains more complete and accurate intersection queuing information by mining GPS data, which provides data for the calculation of dynamic congestion price.

\subsection{Traffic Shockwave Analysis}

This study adopts an estimation model based on the traffic shockwave theory, which can solve the problem of high uncertainty in GPS data. In 1955, British scholars Lighthill and Whitham proposed the fluid dynamics simulation theory of traffic flow (Lighthill \& Whitham, 1955). The theory describes the change in traffic density as traffic shockwaves. When the density of traffic changes, traffic shockwaves will propagate in the traffic flow. The speed of traffic shockwave can be determined by following equation (Eq. (1)) (Lighthill \& Whitham, 1955).

$$
u_{w}=\Delta q / \Delta k=\left(q_{2}-q_{1}\right) /\left(k_{2}-k_{1}\right)
$$


Where $u_{w}$ is the speed of traffic shockwaves, $q_{1}$ and $k_{1}$ are the flow and density of upstream region respectively; $q_{2}$ and $k_{2}$ are the flow and density of downstream region respectively.

As the signal changes, various shockwaves are generated at signalized intersections. These traffic shockwaves interact to form the constant change of the intersection queue. This study defines the effective red light start as the start of a cycle, and the effective green light end as the end of a cycle. In one cycle, queuing shockwaves, discharge shockwaves and departure shockwaves will be formed in sequence. If the queue cannot completely discharge within the effective green time, a residual queue shockwave will be formed (Liu et al., 2009). In the $n t h$ cycle, $w_{1}^{n}$ represents the queuing shockwave, $w_{2}^{n}$ denotes the discharge shockwave, $w_{3}^{n}$ represents the departure shockwave, and $w_{4}^{n}$ is the residual queue shockwave. The speeds of these four shockwaves are represented by $v_{1}^{n}, v_{2}^{n}, v_{3}^{n}$ and $v_{4}^{n}$ respectively.

The queuing shockwave $w_{1}^{n}$ begins to form at the beginning of the effective red and moves upstream of the intersection at speed (Liu et al., 2009).

$$
v_{1}^{n}=\left(0-q_{a}^{n}\right) /\left(k_{j}-k_{a}^{n}\right)
$$

where 0 and $k_{j}$ are the jammed flow and density; and $q_{a}^{n}$ and $k_{a}^{n}$ denote the mean arrival flow rate and density during the $n$th cycle.

The discharge shockwave $w_{2}^{n}$ begins to form at the beginning of the effective green and moves upstream at speed (Liu et al., 2009).

$$
v_{2}^{n}=\left(q_{m}-0\right) /\left(k_{m}-k_{j}\right)
$$

where $q_{m}$ and $k_{m}$ represent the saturation flow and density.

In the real world, the speed of the queuing shockwave $v_{1}^{n}$ is lower than the speed of the discharge shockwave $v_{2}^{n}$. When $w_{1}^{n}$ and $w_{2}^{n}$ meet, the departure shockwave $w_{3}^{n}$ is formed which propagates towards the stop line at speed (Liu et al., 2009).

$$
v_{3}^{n}=\left(q_{m}-q_{a}^{n}\right) /\left(k_{m}-k_{a}^{n}\right)
$$

If the queue cannot be fully discharged in the $n t h$ cycle, a residual queue shockwave $w_{4}^{n}$ will be formed, which propagates upstream from the stop line at a speed $v_{4}^{n}$ (Liu et al., 2009).

$$
v_{4}^{n}=\left(0-q_{m}\right) /\left(k_{j}-k_{m}\right)
$$

The propagation of the above four shockwaves describes the repeated process of queuing formation and dissipation at signalized intersections. Figures 1 and 2 respectively show the propagation process of the four shockwaves. Figure 1 shows the propagation in the undersaturated state, and Fig. 2 shows the propagation in the over- 
Fig. 1 Traffic shockwaves propagation under undersaturation (Liu et al., 2009)

Fig. 2 Traffic shockwaves propagation under over-saturation (Liu et al., 2009)
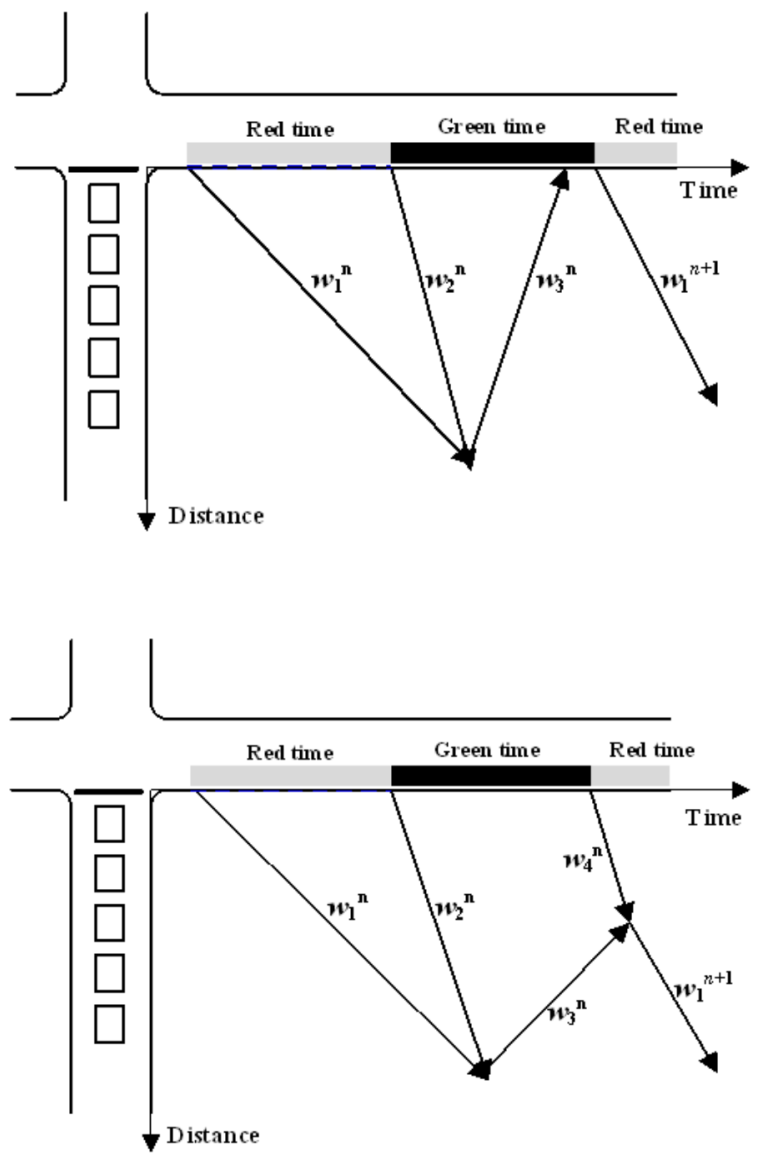

saturated state. If the queue cannot fully discharge in a cycle, the traffic is oversaturated state, otherwise the traffic is undersaturated state.

For an intersection, $q_{m}, k_{m}$ and $k_{j}$ can be regarded as constants, so the values of $v_{2}^{n}$ and $v_{4}^{n}$ can be determined, let $v_{2}^{n}=v_{4}^{n}=v_{f}$, $v_{f}$ is constant.

Among the four shockwaves, the queuing shockwave $w_{1}^{n}$ shows the characteristics of the queue when it is formed. Due to the different arrival rates of vehicles, the queuing shockwaves in different lanes usually have different speeds (Semuel et al., 2018). This study uses the speed $v_{1}^{n}$ of the queuing shockwave as the basis for confirming the lane.

In the same signal cycle, for lanes with the same turn (for example, left turn-lane, through-lane, right-turn, through and right-turn shared lane, etc.), the arrival rates of vehicles and maximum queue lengths are often very similar. For lanes with different turns, the arrival rates and maximum queue lengths are usually quite different. Therefore, this study only focuses on lane identification for lanes with different turns. 


\section{The Research Model and Framework}

\subsection{Real-Time Queuing Shockwave Speed Estimation}

This research mainly studies the general situation of queuing at intersections and the assumptions about the model are as follows:

(1) The study subject is a given intersection, and the signal timing information is known.

(2) In one cycle, the arrival rate is regarded as a constant, which can be considered as the average arrival rate of the cycle. For different cycles, the arrival rates are variable.

(3) The waiting time of any vehicle at the intersection cannot exceed two cycles.

(4) In each cycle and each queue, at least one queued vehicle can provide the realtime GPS data.

According to Gazis's definition (Gazis, 1964) of the over-saturated state of the intersection, this study gives the definition of the cycle state. If the queue can discharge completely within the effective green time of a cycle, the cycle is undersaturation. If the queue cannot all discharge during the effective green time of a cycle and a residual queue is formed, then the cycle is defined as over-saturation.

\section{$A$. Queuing delay analysis.}

The queuing time of a vehicle refers to the time span from the time when the vehicle first stops in line to the time when the vehicle pass the stop bar. The queuing delay of vehicles refers to the time difference between the queuing time of vehicles and the time that vehicles pass through the intersection at free flow speed. According to the reasons for the delays, this study divides queuing delays into four parts, which are defined as phase delays, discharge delays, over-saturation delays and random delays.

- Phase delay: the waiting time for the green light. The phase delay is related to the time when the vehicle arrives at the intersection and the state of the phase at that time. $D_{p}$ represents the phase delay of vehicle $A$.

- Discharge delay: the delay caused by the discharging process of the queue, that is, the propagation time of the discharge shockwave $w_{2}^{n} . D_{s}$ represents the discharge delay of vehicle $A$.

- Over-saturation delay: the delay caused by the over-saturated traffic conditions. During the over-saturated cycle, the vehicles in the residual queue will not pass the stop line until the effective green time of the next cycle. The Over-saturation delay is equal to the effective red time of the next cycle. $D_{e}$ represents the oversaturation delay of vehicle $A$. This study defines vehicles with $D_{e}\left(D_{e}>0\right)$ as over-saturated vehicles.

- Random delay: the extra delays caused by the vehicles themselves and the environment. For example, the start-up process is slow due to the vehicle type and the driver's reaction, or the delay caused by the distance between vehicle $A$ and the vehicle ahead is less than the safe distance. In general, the starting time of a vehicle is used as a parameter to calculate the effective green time in this study. $D_{r}$ represents the random delay of vehicle $A$. 
Figure 3 illustrates the phase delay, the discharge delay and the over-saturation delay using traffic shockwaves and vehicle trajectories. $T_{r}^{n}$ and $T_{g}^{n}$ respectively represent the start time of the effective red and the effective green in the $n$th signal cycle.

Among these four types of delays, only the discharge delay is directly related to the position of the vehicle in the queue. Using the vehicle's discharge delays, the queuing shockwave velocity $v_{1}^{n}$ can be estimated in real time.

\section{$B$. Queuing shockwave speed estimation algorithm.}

The model in this paper uses the vehicle's stopping time and the phase change data to calculate the vehicle's discharge delays, and then further estimates the queuing shockwave speed based on the discharge delays. The calculation of the stopping time is related to the definition of the intersection range. stopping within the intersection area is regarded as stopping due to queuing. The intersection range given by most researchers is between $100 \mathrm{~m}$ and $200 \mathrm{~m}$ (Dong \& Zhou, 2020). This study believes that the range of the intersection is difficult to determine with a unified standard, and the range needs to be specified respectively according to the road conditions and traffic states at the intersection.

As indicated in Fig. 3, the stopping time of a vehicle includes phase delay and discharge delay. For over-saturated vehicles, stopping time also includes over-saturation delay. Random delay may exist during the entire process of vehicle stopping and moving. $T_{d}$ represents the total stopping time of vehicle $A$ within the range of the intersection. This algorithm only analyze the GPS data of vehicles with $T_{d}$ greater than 0 .

The algorithm includes the following steps:

Step 1. Calculate the over-saturation delay of vehicle $A$.

The first step of the algorithm is to determine whether vehicle $A$ is an over-saturated vehicle. When vehicle $A$ meets the following conditions, the state of the vehicle is over-saturated.

$$
T_{r}^{n} \leqslant t \leqslant T_{r}^{n+1} \text { and } T_{l} \geqslant T_{g}^{n+1}
$$

$t$ represents the time when vehicle $A$ first stopped at the intersection. $T_{l}$ is the last time vehicle $A$ started within the intersection range. The left part of Eq. (6) indicates that vehicle $A$ arrives at the intersection in the $n t h$ cycle and starts to queue up, and

Fig. 3 Queuing delay under over-saturation

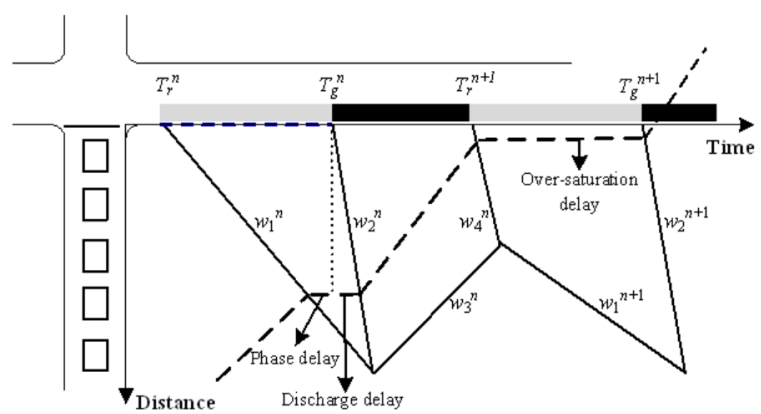


the right part of Eq. (6) indicates that vehicle $A$ leaves the intersection at the effective green time of the $n+1$ th cycle. So the over-saturation delay of vehicle $A$ is

$$
D_{e}=\left\{\begin{array}{cc}
0, & \text { if } T_{r}^{n} \leqslant t \leqslant T_{r}^{n+1} \text { and } T_{l}<T_{r}^{n+1} \\
T_{g}^{n+1}-T_{r}^{n+1}, & \text { if } T_{r}^{n} \leqslant t \leqslant T_{r}^{n+1} \text { and } T_{l} \geqslant T_{g}^{n+1}
\end{array}\right.
$$

Step 2. Calculate the phase delay of vehicle $A$

$$
D_{p}=\left\{\begin{array}{l}
0, \quad \text { if } T_{g}^{n} \leqslant t \leqslant T_{r}^{n+1} \\
T_{g}^{n}-t, \quad \text { if } T_{r}^{n} \leqslant t<T_{g}^{n}
\end{array}\right.
$$

Step 3. Estimate the random delay of vehicle $A$.

If vehicle $A$ is a bus or other large vehicle, the random delay of $A$ is increased by $1 \mathrm{~s}$. In addition, if the distance between $A$ and the vehicle ahead can be obtained, and the distance is less than $1 \mathrm{~m}$, the random delay of $A$ will increase by $1 \mathrm{~s}$. In other cases, the random delay of vehicle $A$ is 0 . Since the market penetration rate cannot be determined, the random delays of vehicles can only be estimated based on available data. As the market penetration rate increases, the estimation of random delays will be more accurate.

Step 4. Calculate the discharge delay of vehicle $A$

$$
D_{s}=T_{d}-D_{p}-D_{e}-D_{r}
$$

Step 5. Estimate the queuing shockwave speed.

$T_{s}$ is the time for the discharge shockwave $w_{2}^{n}$ to propagate from the stop bar to vehicle $A$.

$$
T_{s}=\left\{\begin{array}{cc}
D_{s}, & \text { if } T_{r}^{n} \leqslant t \leqslant T_{g}^{n} \\
D_{s}+\left(t-T_{g}^{n}\right), & \text { if } T_{g}^{n}<t \leqslant T_{r}^{n+1}
\end{array}\right.
$$

$L_{A}$ indicates the queue length of vehicle $A$, that is, the distance from the stop bar to $A$ when vehicle $A$ stops for the first time. According to the previous analysis, this equation can be derived: $L_{A}=-T_{S} * v_{2}^{n}=-T_{S} * v_{f}$. $v_{f}$ can be calculated based on the saturation flow rate $q_{m}$, the saturation density $k_{m}$, and the jammed density $k_{j}$. The queuing shockwave speed can be calculated as in the following equation:

$$
v_{1}^{n}=\frac{L_{A}}{t-T_{r}^{n}}=\frac{T_{s} * v_{f}}{T_{r}^{n}-t}
$$

When the market penetration rate increases, more and more queued vehicles can provide GPS data, and the accuracy of $v_{1}^{n}$ estimation will improve.

\subsection{Lane Identification Algorithm}

As mentioned above, for lanes with different turns at intersections, the speeds of the queuing shockwaves are usually different. This study uses classifying and clustering 
methods to estimate the queuing shockwave speeds of lanes with different turns, so as to identify the lane where the queue is located. Because this paper only clusters queuing shockwave speeds based on similarity, the less complex classification and clustering methods are chosen. The real-time performance of these two algorithms is very good and can meet the requirements for real-time lane identification.

\section{$\boldsymbol{A}$. KNN classifying method.}

$\mathrm{KNN}$ (k-nearest neighbor) is a supervised learning method. In this study, the speeds of queuing shockwaves are used as one-dimensional variables of KNN clustering.

The inputs of the method includes:

(1) Training dataset.

$T=\left\{\left(x_{1}^{j}, y_{1}\right),\left(x_{2}^{j}, y_{2}\right),\left(x_{3}^{j}, y_{3}\right), ?,\left(x_{i}^{j}, y_{i}\right), ?,\left(x_{n}^{j+m}, y_{n}\right)\right\}, j, i, n, m>0, x_{i}^{j}$ is the speed of the queuing shockwave in the $j$ th cycle, $y_{i}$ is the category, $y_{i} \in\left\{c_{1}, c_{2}\right\} . c_{1}$ and $c_{2}$ indicate left turn lanes and through lanes respectively.

(2) Clustering object $x$.

(3) The number of nearest neighbors $k$.

The output of this method is the category to which $x$ belongs.

Step 1. According to a given distance threshold, find the $k$ points closest to the estimated object $x$ in $T$, and the neighborhood of $x$ is denoted as $N_{k}(x)$.

Step 2. Among these $k$ points, determine the category $y$ of $x$ according to the weighted voting method.

$$
y=\left\{\begin{array}{c}
\arg \max _{c_{z}} \sum_{x_{i}^{\beta} \in N_{k}(x)} \frac{c_{z}}{\left(x_{i}^{\beta}-x\right)^{2}} \\
y_{i} \quad x_{i}^{\beta}=x
\end{array} \quad x_{i}^{\beta} \neq x, z=1,2, \beta>0\right.
$$

\section{B. DBSCAN Clustering Method.}

DBSCAN (Density-based spatial clustering of applications with noise) is a clustering algorithm based on spatial density. It can be used to divide clusters of any shape and is not sensitive to noise. But the traditional algorithm is not suitable for the queuing shockwave speed clustering. This paper has modified the traditional DBSCAN algorithm, and the steps of the modified DBSCAN algorithm are described as follows.

The inputs of the method includes:

(1) The training dataset $T$ is divided into two sub datasets, $T_{c 1}$ and $T_{c 2}$, according to their categories.

, ,

$T_{c 2}=\left\{\left(x_{n}^{j}, c_{2}\right),\left(x_{n+1}^{j}, c_{2}\right),\left(x_{n+2}^{j}, c_{2}\right), ?,\left(x_{n+m}^{j+m}, c_{2}\right)\right\}, k, j, q, n, m>0 . x_{i}^{\beta}(i, \beta>0)$ is the speed of the queuing shockwave in the $\beta$ th cycle, and $T_{c 1}$ and $T_{c 2}$ are the datasets belonging to $c_{1}$ (left turn lane) and $c_{2}$ (through lane) categories, respectively.

(2) Clustering object dataset $D$.

(3) The radius $\varepsilon$.

(4) Density threshold MinPts.

The output of this method is the clustering result of dataset $D$.

Step 1. Search for points that meet the threshold MinPts requirements in an $\varepsilon$-radius of $T_{c 1}$ and $T_{c 2}$ respectively. Take these points as the core points, denoted as $\left\{P_{c 1}\right\},\left\{P_{c 2}\right\}$. 
Step 2. Traverse the dataset $D$, find all the density-reachable points from $\left\{P_{c l}\right\}$, $\left\{P_{c 2}\right\}$ respectively, and update the clusters $\left\{P_{c 1}\right\}$ and $\left\{P_{c 2}\right\}$.

Step 3. Generate the final cluster result through density-connected points.

Step 4. Repeat step 2 and step 3 until all points in dataset $D$ are processed.

Step 5. Compute the cluster centers $e_{1}$ and $e_{2}$ in $T_{c 1}$ and $T_{c 2}$ respectively.

$$
\begin{aligned}
& e_{1}=\arg \lim _{T_{1}} \sum\left(\left|x_{e 1}-x_{i}^{\beta}\right|^{2}\right) \\
& e_{2}=\arg \lim _{T_{2}} \sum\left(\left|x_{e 2}-x_{i}^{\beta}\right|^{2}\right)
\end{aligned}
$$

Step 6. For noise data not included in any clusters and overlapping data in two clusters, determine the clusters they belong to according to their Euclidean distances from $e_{1}$ and $e_{2}$.

If the queuing shockwave speeds of lanes with different turns are significantly different, the algorithm in this study can be used to identify lanes. However, if the shockwave speeds between lanes of different turns are close, it means that the arrival rates of these lanes are similar. In this case, the queue lengths of different lanes can be calculated based on the signal timing information. Although the algorithms in this study only determine the two sets of lanes with different turning, these algorithms are also applicable if other turning lanes are added.

\subsection{Real-Time Queue Length Estimation Model}

This section discusses how to use GPS data to estimate the real-time queue length at signalized intersections.

\section{$A$. Undersaturated state.}

As Sect. 3 described, when the queuing shockwave $w_{1}^{n}$ meets the discharge shockwave $w_{2}^{n}$, the departure shockwave $w_{3}^{n}$ is formed, and the queuing length reaches the maximum at the same time. $T_{\max }^{n}$ is the time of maximum queue achieved during $n$th cycle. When the $n$ - 1 th cycle is undersaturated, the maximum queue length $L_{\max }^{n}$ ( $m$ ) during $n t h$ cycle is:

$$
L_{\max }^{n}=-v_{1}^{n}\left(T_{\max }^{n}-T_{r}^{n}\right)=-v_{2}^{n}\left(T_{\max }^{n}-T_{g}^{n}\right)
$$

Equation (15) can be further derived as in the following equation:

$$
T_{\max }^{n}=\frac{v_{1}^{n} T_{r}^{n}-v_{2}^{n} T_{g}^{n}}{v_{1}^{n}-v_{2}^{n}}
$$

Then $L_{\max }^{n}$ can be calculated:

$$
L_{\max }^{n}=\frac{v_{1}^{n} v_{f}}{v_{1}^{n}-v_{f}}\left(T_{g}^{n}-T_{r}^{n}\right)
$$


where $v_{f}$ is constant, $v_{1}^{n}$ can be calculated by Eq. (11).

\section{$B$. Over-saturated state.}

When the previous cycle is oversaturated, the maximum queue length is not only formed by the vehicles arriving in this cycle, but also affected by the residual queue of the previous cycle.

If $n$th cycle is oversaturated, when the departure shockwave $w_{3}^{n}$ and the residual queue shockwave $w_{4}^{n}$ meet in $n+1$ th cycle, the residual queue length is maximized. $T_{\text {res }}^{n}$ is the time when the residual queue length reaches the maximum in $n t h$ cycle, so that:

$$
v_{3}^{n}\left(T_{r e s}^{n}-T_{r}^{n+1}\right)-v_{f}\left(T_{r e s}^{n}-T_{r}^{n+1}\right)=L_{\max }^{n}-v_{3}^{n}\left(T_{r}^{n+1}-T_{\max }^{n}\right)
$$

Equation (18) can be rewritten:

$$
T_{\text {res }}^{n}=\frac{v_{3}^{n} T_{\max }^{n}-v_{f} T_{r}^{n+1}+L_{\max }^{n}}{v_{3}^{n}-v_{f}}
$$

$L_{\text {res }}^{n}$ represents the maximum residual queue length during $n t h$ cycle. Since $T_{r e s}^{n}=T_{r}^{n+1}-L_{r e s}^{n} / v_{f}, L_{r e s}^{n}$ can be calculated in the following equation:

$$
L_{\text {res }}^{n}=\frac{v_{3}^{n}\left(T_{\max }^{n}-T_{r}^{n+1}\right)+L_{\max }^{n}}{v_{f}-v_{3}^{n}} v_{f}
$$

When the $n$ - 1 th cycle is over-saturated, the maximum queue length during $n t h$ cycle is:

$$
L_{\max }^{n}=-v_{1}^{n}\left(T_{\max }^{n}-T_{\text {res }}^{n-1}\right)+L_{\text {res }}^{n-1}=-v_{2}^{n}\left(T_{\max }^{n}-T_{g}^{n}\right)
$$

$T_{\max }^{n}$ can be expressed as:

$$
T_{\max }^{n}=\frac{v_{1}^{n} T_{r}^{n}-v_{f} T_{g}^{n}}{v_{1}^{n}-v_{f}}-\frac{L_{r e s}^{n-1}}{v_{f}}
$$

Then the maximum queue length $L_{\max }^{n}$ in $n t h$ cycle can be estimated by:

$$
L_{\max }^{n}=\frac{v_{1}^{n} v_{f}}{v_{1}^{n}-v_{f}}\left(T_{g}^{n}-T_{r}^{n}\right)+L_{r e s}^{n-1}
$$

Using the queuing shockwave speed $v_{1}^{n}$ and the vehicle speed, the arrival traffic state $\left(q_{a}^{n}, k_{a}^{n}\right)$ can be estimated, and then the departure shockwave speed $v_{3}^{n}$ can be calculated according to Eq. (4). According to Eq. (20) and Eq. (23), the maximum queue length $L_{\max }^{n}$ and the maximum residual queue length $L_{r e s}^{n}$ in the over-saturated $n t h$ cycle can be finally estimated. 


\section{Experimental Design and Performance Evaluation}

The proposed models are applied to the intersection of Tianhe North Road and Tianshou Road in Guangzhou, China. As shown in Fig. 4. Tianhe North Road is a main road in the central business district of Guangzhou. The intersection of Tianhe North Road and Tianshou Road is often a congested intersection, and there is a large difference in queue length between through lane and left-turn lane. The intersection is suitable to apply the model proposed in this paper.

This study used 8 vehicles equipped with GPS equipment as test vehicles. At the intersection of Tianhe North Road, there are 6 lanes from west to east, including 2 left-turn lanes, 3 through lanes, and 1 right-turn lane, which are marked as lane 1-6 from the inside to the outside. Lane 1-2 are left-turn lanes, and lane 3-5 are through lanes. According to the analysis result of the traffic flow at the intersection, the intersection range is a circular area with the center of the intersection as the center and a radius of $240 \mathrm{~m}$.

Fig. 4 Study site in Guanzhou: tianhe north road-tianshou road

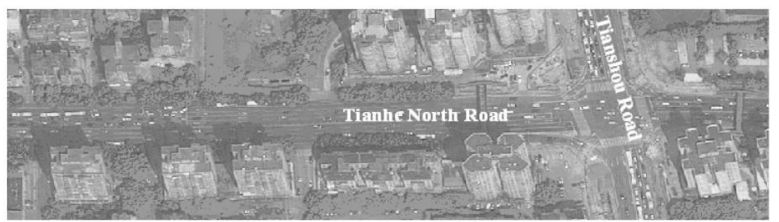

Fig. 5 Implementation flow chart

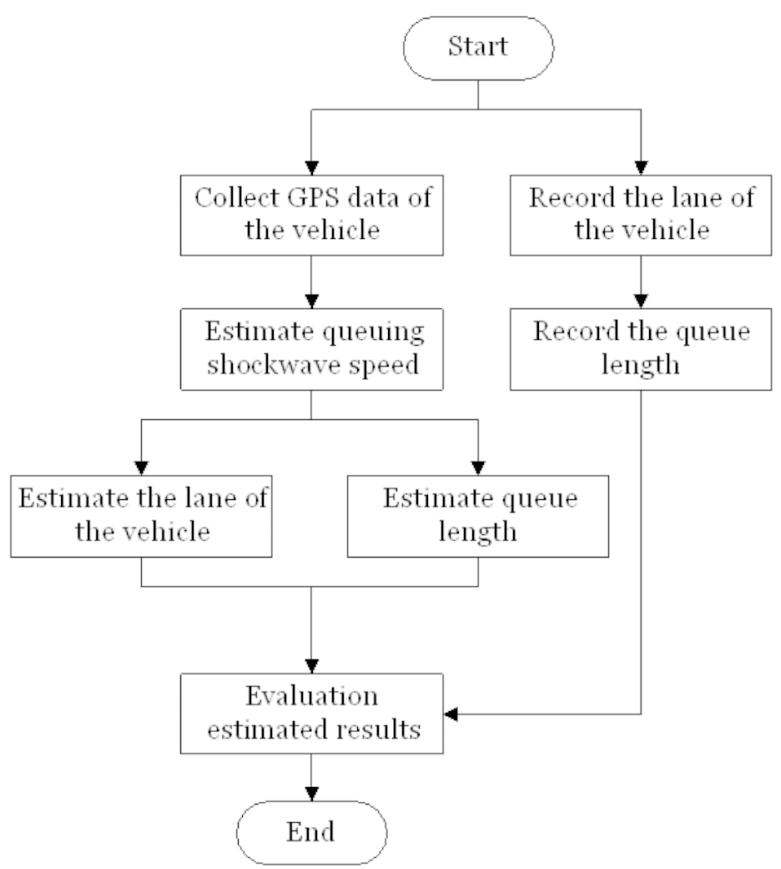


This study tested lane 1-4 separately. In each test period, 8 vehicles selected the same lane of Tianhe North Road to pass through the intersection as required. Four cameras were deployed at the intersection to record the real queuing situation.

The test time is the evening of November 20, 2019 and November 24, 2019. The test period selected is evening peaks, and each test takes about $2 \mathrm{~h}$. These two experiments are denoted Experiment 1 (November 20, 2019) and Experiment 2 (November 24,2019$)$ respectively. On this test road, the saturation flow is $1780 \mathrm{veh} / \mathrm{h} / \mathrm{lane}$, and the jammed density is $124 \mathrm{veh} / \mathrm{km} / \mathrm{lane}$. During the test, the test vehicles were driving on the designated route, which cannot guarantee that there are one or more vehicles queuing at the intersection in each cycle. Therefore, for the cycles that meet the test requirements, there may be insufficient data in the previous cycle. If the previous cycle is in an undersaturated state, insufficient data has no effect on the test results. If the previous cycle is over-saturation and insufficient data, this study used the observed value of the residual queue length instead of the estimated value as the input data of the test cycle. This has little effect on the test results.

Figure 5 is the implementation flow chart of the experiment.

\subsection{Shockwave Speed Estimation Analysis}

On November 24, 2019 (Sunday), the test started at 17:03 and ended at 19:06, including 46 cycles. Due to insufficient data, 18 cycles cannot be estimated. This paper sets the ID of the cycle starting at 17:03:16 as 1. Table 1 shows the comparison between the estimated results and the measured data from 18:04 to 18:29.

In Table 1, Column 1 shows the record sequence number. Column 2 shows the ID of the cycle. Column 3 specifies the Lane. Lane 1 is left-turn lane and lane 3 is through lane. Column 4 shows the number of valid test vehicles. An valid test vehicle is a probe vehicle that stopped at the intersection in the lane during the cycle. Column

Table 1 Queuing shockwave speed estimation results (2019/11/24 18:04-18:29)

\begin{tabular}{llllllr}
\hline Record ID & Cycle ID & Lane & $\begin{array}{l}\text { Number of valid } \\
\text { test vehicles } \\
(\text { veh })\end{array}$ & $\begin{array}{l}\text { Measured } \\
\text { shockwave } \\
\text { speed }(\mathrm{km} / \mathrm{h})\end{array}$ & $\begin{array}{l}\text { Estimate shock- } \\
\text { wave speed } \\
(\mathrm{km} / \mathrm{h})\end{array}$ & $\begin{array}{l}\text { Rela- } \\
\text { tive } \\
\text { error } \\
(\%)\end{array}$ \\
\hline 1 & 15 & 1 & 2 & 4.41 & 3.86 & 12.34 \\
2 & 17 & 1 & 1 & 4.67 & 5.55 & 18.69 \\
3 & 19 & 1 & 3 & 4.43 & 3.80 & 14.20 \\
4 & 20 & 1 & 1 & 4.89 & 3.86 & 21.06 \\
5 & 22 & 1 & 4 & 4.65 & 4.95 & 6.36 \\
6 & 24 & 1 & 2 & 4.30 & 5.06 & 17.67 \\
7 & 26 & 3 & 3 & 7.10 & 6.39 & 10.01 \\
8 & 27 & 3 & 3 & 6.30 & 7.07 & 12.20 \\
9 & 29 & 3 & 1 & 6.96 & 7.99 & 14.78 \\
10 & 31 & 3 & 1 & 6.71 & 5.33 & 20.56 \\
11 & 33 & 3 & 2 & 7.47 & 8.35 & 11.71 \\
12 & 35 & 3 & 4 & 6.58 & 7.35 & 11.67 \\
\hline
\end{tabular}


5 shows the measured queuing shockwave speed. Column 6 shows the estimated queuing shockwave speed. Columns 7 reports the relative error.

Table 2 shows the Mean Absolute Percentage Error (MAPE) of queuing shockwave speed. The higher MAPE is $15.00 \%$, which can meet the requirements for lane identification.

Figure 6 shows the relationship between the relative error of shockwave speed estimation and the number of valid test vehicles. The solid line in Fig. 6 is the logarithmic trend line, and the fitting formula is $y=-0.068 \ln (x)+0.1887$. When the number of valid test vehicles increases from 1 to 6 , the MAPE drops from $18.60-6.58 \%$. When the number of valid test vehicles is greater than 2, the downward trend of MAPE is more significant. Test results show that the more test vehicles are valid, the more accurate the algorithm estimation is

Vehicles.

This paper also performed experiments in the two intersections of Guangzhou, namely the intersection of Tianhe North Road and Linhe East Road, the intersection of Tianhe Road and Sports East Road. The concerning data of the two experiments is shown in Table 3.

Table 2 MAPE of shockwave speed estimation in Experiment 1,2

\begin{tabular}{llll}
\hline Experiment ID & Test time & Number of test cycles & MAPE (\%) \\
\hline Experiment 1 & $2019 / 11 / 2017: 10-19: 12$ & 26 & 15.00 \\
Experiment 2 & $2019 / 11 / 2417: 03-19: 06$ & 28 & 14.24 \\
\hline
\end{tabular}

Fig. 6 Relative error of estimated shockwave speed vs. valid test

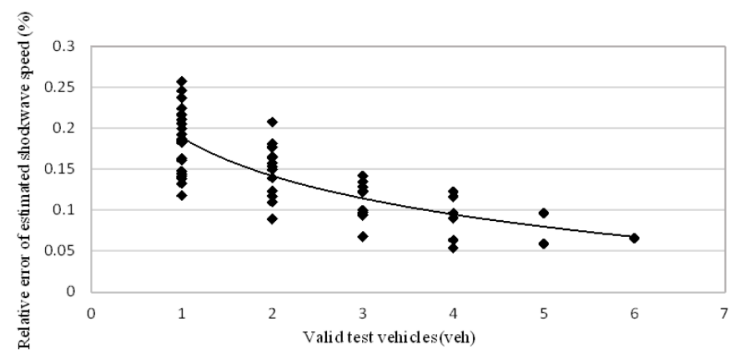

Table 3 Experiment 3 and Experiment 4 related data

\begin{tabular}{llrl}
\hline Experiment ID & Site & \multicolumn{1}{l}{ Test time } & Number of test cycles \\
\hline Experiment 3 & Tianhe North Road-Linhe East Road & $2020 / 5 / 307: 41-9: 25$ & 20 \\
Experiment 4 & Tianhe Road-Sports East Road & $2020 / 5 / 27$ 17:26-19:20 & 23 \\
\hline
\end{tabular}

Table 4 MAPE of shockwave speed estimation Experiment 3, 4

\begin{tabular}{llll}
\hline Experiment ID & Test time & Number of test cycles & MAPE (\%) \\
\hline Experiment 3 & $2020 / 5 / 307: 41-9: 25$ & 20 & 14.53 \\
Experiment 4 & $2020 / 5 / 2717: 26-19: 20$ & 23 & 14.87 \\
\hline
\end{tabular}


The MAPE of queuing shockwave speed in Experiments 3 and 4 is shown in Table 4

Although due to traffic conditions, the data collected in Experiments 3 and 4 is not much, the estimation accuracy of shockwave speed is also satisfactory.

\subsection{Shockwave Speed Classification and Cluster Analysis}

In this study, the estimated data of the two tests were classified and clustered. The estimated data set of Experiment 1 is recorded as $D_{1}$, and the estimated data set of Experiment 2 is recorded as $D_{2}$. The first 5 sets of observational data of lane 1 and lane 3 are used as the training data in each test.

(1) KNN.

In applications, $k$ is often set to a low number. According to $k$-foldcross validation, this study set $k=5$. The accuracies of lane identification is $84.62 \%$ (dataset $D_{1}$ ) and $92.86 \%$ (data set $\left.D_{2}\right)$.

(2) DBSCAN.

By means of k-distance graph (set $\mathrm{k}=4$ ), the radiuses of the two datasets can be obtained, which are 0.48 (dataset $D_{1}$ ) and 0.42 (data set $D_{2}$ ) respectively. According to the characteristics of DBSCAN, the value of the density threshold MinPts needs to be equal to or greater than 3. If MinPts is too high, two adjacent clusters may be merged into one cluster. This study set MinPts $=4$. For dataset $D_{1}(\varepsilon=0.48$, MinPts $=4)$ the accuracy of lane identification is $84.62 \%$. For dataset $D_{2}(\varepsilon=0.42$, MinPts $=4$ ) the accuracy of lane identification is $96.43 \%$.

The results show that, the estimation accuracies of the two methods are high, but the estimation of DBSCAN is more accurate. The reason for the high estimation accuracy is that there is a large difference in traffic flow between the two sets of lanes, and the speeds of the queuing shockwaves hardly overlap. The measured shockwave speed values of the two tests are shown in Fig. 7.

In Fig. 7, the dot $(\bullet)$ is the shockwave speed in the left-turn lane, and the triangle point $(\pi)$ is the shockwave speed in the through lane. The average shockwave speeds of the left-turn lane are $4.90 \mathrm{~km} / \mathrm{h}$ (Experiment 1) and $4.61 \mathrm{~km} / \mathrm{h}$ (Experiment 2), and the average shockwave speeds of the through lane are $7.92 \mathrm{~km} / \mathrm{h}$ (Experiment 1 ) and $7.89 \mathrm{~km} / \mathrm{h}$ (Experiment 2). The difference between the average values of the two sets of lanes is $3.02 \mathrm{~km} / \mathrm{h}$ (Experiment 1) and $3.28 \mathrm{~km} / \mathrm{h}$ (Experiment 2).
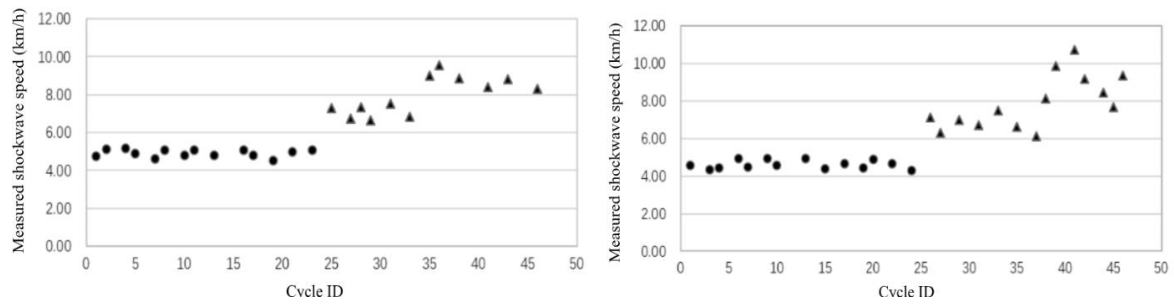

Fig. 7 Illustration of measured shockwave speeds. (a) Experiment 1 (b) Experiment 2 
The queuing shockwave speeds of lanes with different turns are quite different, which is very common in urban road networks. In this case, the precision of the lane identification is good. However, if the queuing shockwave speeds of lanes with different turns are similar, the precision of lane prediction will decrease. In those situations, since queuing shockwave speeds are very close, the queue length can be calculated according to the effective green time of the lane, so the lane-based realtime queue length estimation will be easier.

The lane identification accuracies of Experiments 1-4 are shown in Table 5.

At present, there are few studies on lane identification based on GPS data. Rompis et al. (Semuel et al., 2018) validated the lane identification algorithm using data generated from simulation models. In (Semuel et al., 2018), when the probability threshold (the probability where provide enough cycles for all market penetration rates) is $85 \%$, the accuracy prediction is $85 \%$ or over. As mentioned above, the method is only suitable for undersaturated traffic. This paper tested the lane identification algorithm with real field data, and there are no strict conditions for the algorithm application. The accuracy of the proposed algorithm is above $82.34 \%$, so the lane can be identified with acceptable results. In addition, the proposed algorithm is suitable for undersaturated and over-saturated traffic.

Table 5 Accuracies of lane identification

\begin{tabular}{lll}
\hline Experiment ID & KNN & DBSCAN \\
\hline Experiment 1 & $84.62 \%$ & $84.62 \%$ \\
Experiment 2 & $92.86 \%$ & $96.43 \%$ \\
Experiment 3 & $82.34 \%$ & $82.34 \%$ \\
Experiment 4 & $90.76 \%$ & $94.35 \%$ \\
\hline
\end{tabular}

Table 6 Queue length estimation results (18:04-18:29)

\begin{tabular}{llllllr}
\hline Record ID & Cycle ID & Lane & $\begin{array}{l}\text { Number of valid } \\
\text { test vehicles } \\
\text { (veh) }\end{array}$ & $\begin{array}{l}\text { Measured } \\
\text { queue length } \\
(\mathrm{m})\end{array}$ & $\begin{array}{l}\text { Estimated queue } \\
\text { length }(\mathrm{m})\end{array}$ & $\begin{array}{l}\text { Rela- } \\
\text { tive } \\
\text { error } \\
(\%)\end{array}$ \\
\hline 1 & 15 & 1 & 2 & 170.4 & 153.58 & 9.87 \\
2 & 17 & 1 & 1 & 187.7 & 199.84 & 6.47 \\
3 & 19 & 1 & 3 & 174.4 & 162.82 & 6.64 \\
4 & 20 & 1 & 1 & 182.4 & 157.53 & 13.63 \\
5 & 22 & 1 & 4 & 170.2 & 185.87 & 9.21 \\
6 & 24 & 1 & 2 & 177.6 & 198.38 & 11.70 \\
7 & 26 & 3 & 3 & 82.8 & 74.51 & 10.01 \\
8 & 27 & 3 & 3 & 73.5 & 82.47 & 12.20 \\
9 & 29 & 3 & 1 & 81.2 & 93.2 & 14.78 \\
10 & 31 & 3 & 1 & 78.3 & 62.2 & 20.56 \\
11 & 33 & 3 & 2 & 87.2 & 97.41 & 11.71 \\
12 & 35 & 3 & 4 & 76.8 & 85.76 & 11.67 \\
\hline
\end{tabular}




\subsection{Queue Length Estimation Analysis}

Table 6 shows results comparing the estimated values and the measured data of the queue length from 18:04 to 18:29 on November 24, 2019 (Experiment 2).

In Table 6, Column 1 shows the record sequence number. Column 2 shows the ID of the cycle. Column 3 specifies the Lane. Column 4 shows the number of valid test vehicles. Column 5 shows the measured maximum queue length. Column 6 shows the estimated maximum queue length. Columns 7 reports the relative error

Figure 8 shows the relationship between the relative error of the estimated queue length and the number of valid test vehicles. The solid line in Fig. 8 is the logarithmic trend line, and the fitting formula is $\mathrm{y}=-4.875 \ln (\mathrm{x})+15.44$. When the number of valid test vehicles increases from 1 to 6 , the MAPE of estimated queue length drops from $15.68-6.88 \%$. When the number of valid test vehicles is greater than 1 , the decreasing trend of MAPE is more obvious. The experimental results show that, as the number of valid test vehicles increases, the accuracy of the queuing length estimation becomes higher and higher

Table 7 shows the MAPE of the queue length estimation in Experiments 1-4.

In the MAPE of these four experiments, the highest MAPE is $12.40 \%$, which is less than $25.49 \%$ of the field test result in (Cheng et al., 2011). With the same number of sampled vehicles, the proposed model has higher prediction accuracy than the method in reference (Tan et al., 2021)

\section{Conclusions}

The Covid-19 pandemic has caused a significant reduction in the use of public transport worldwide. Therefore, more private car trips have caused more serious con-

Fig. 8 Relative error of estimated queue length vs. valid test vehicles

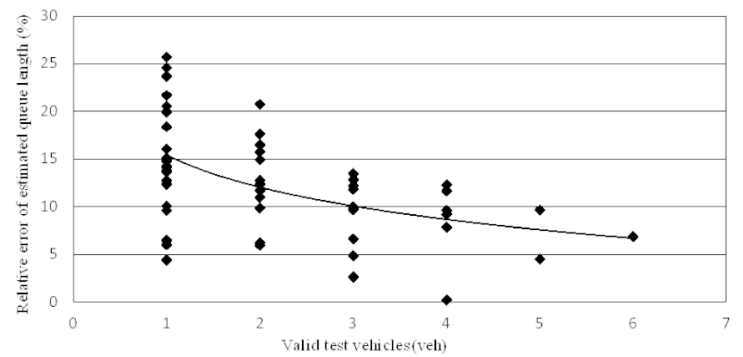

Table 7 MAPE of queue length estimation

\begin{tabular}{lll}
\hline Experiment ID & Number of test cycles & MAPE (\%) \\
\hline Experiment 1 & 26 & 12.34 \\
Experiment 2 & 28 & 12.40 \\
Experiment 3 & 20 & 12.27 \\
Experiment 4 & 23 & 12.38 \\
\hline
\end{tabular}


gestion in many cities. Congestion charging can reduce traffic congestion. A lot of congestion taxes will be used to improve public transport, so congestion charging will make a significant contribution to financial markets. How to monitor traffic congestion at low cost is pressing issue in the implementation of congestion charging. In the era of big data, AI algorithm based on massive GPS data are a promising solution to this problem.

This paper proposed an innovative approach to identify the lanes of queues and estimate the queue length at signalized intersection using real-time GPS data. The proposed methods were tested with field data in Guangzhou, China. The accuracy of lane estimation is above $82.34 \%$. The accuracy of the queue length estimation model is satisfactory. The results show that the model can produce satisfactory real-time queuing information for congestion charging.

There are research limitations and some possible research directions for the future works:

The proposed model requires high densely sampled GPS data with a frequency of $1 \mathrm{~s}$. At present, a large amount of data cannot meet this requirement, and the pulling frequency is $3 \mathrm{~s}$ or more. Therefore, the estimation of the queue based on sparsely sampled GPS data can be further studied in the future.

The accuracy of the estimation model depends on the number of sampling vehicles. If there is less than one queued sampling vehicle, the lane and queue length cannot be calculated. If the historical GPS data can be used to supplement the lack of data, the problem of insufficient data will be solved. How to apply the historical data while maintaining real-time performance is another issue to be studied in the future.

Some vehicles did not stop at the intersection, but their trajectory data is still closely related to the queue length at intersection. The regularity of the trajectory data can be mined in future research.

Link travel time, vehicle delays, the distance travelled, etc. are also basic data for congestion charging. In the future, it is necessary to study the complete GPS data solution to monitor traffic and vehicle running status for congestion charging.

Author Contribution Yu Wang; conceptualization and interpretation of the results in the context. Dongbo zhang; data curation and interpretation of the results in concerns regarding GPS Data. Yu Zhang; analytic techniques, data importation, computing. All authors read and approved the final manuscript.

Funding This work was supported by the open fund for the Key Laboratory for traffic and transportation security of Jiangsu Province, China (No. TTS2016-03), Industry University Research Cooperation Project of Jiangsu Province, China (No. BY2020050), the Natural Science Foundation of Guangdong Province under Grant 2021A1515012529, and GDAS' Special action for building a first-class research institution in China 2020GDASYL-20200402007.

\section{Ethical Approval}

Conflict of Interest The authors declare no conflict of interest/competing interest. 


\section{References}

An, C., Wu, Y. J., Xia, J., \& Huang, W. (2017). Real-time queue length estimation using event-based advance detector data. Journal of Intelligent Transportation Systems, 277-290. https://doi.org/10.10 $80 / 15472450.2017 .1299011$

Bocarejo, J. P. (2020). Congestion in Latin American Cities: Innovative Approaches for a Critical Issue[C]// International Transport Forum Discussion Papers. OECD Publishing, 9. https://doi. org/10.1787/938de08e-en

Buckingham, C., Doherty, A. R., Hawkett, D., et al. (2010). Central London congestion charging: understanding its impacts[J]. Transport, 163(2), 73-83. https://doi.org/10.1680/tran.2010.163.2.73

Cai, Q., Wang, Z., Zheng, L., \& Wu, B. (2014). Shock wave approach for estimating queue length at signalized intersections by fusing data from point and mobile sensors. Transportation Research Record Journal of the Transportation Research Board, 2422(2422), 79-87. https://doi.org/10.3141/2422-09

Cheng, Y., Qin, X., Jin, J., et al. (2011). Cycle-by-Cycle Queue Length Estimation for Signalized Intersections Using Sampled Trajectory Data[J].Transportation Research Record Journal of the Transportation Research Board, 2257(1):87-94. https://doi.org/10.3141/2257-10.

Cipriani, E., Mannini, L., Montemarani, B., et al. (2019). Congestion pricing policies: Design and assessment for the city of Rome, Italy[J]. Transport Policy, 80(AUG.), 127-135. https://doi.org/10.1016/j. tranpol.2018.10.004

Dong, S., \& Zhou, J. (2020). A Comparative Study on Drivers' Stop/Go Behavior at Signalized Intersections Based on Decision Tree Classification Model[J]. Journal of Advanced Transportation, 2020 (2):1-13. https://doi.org/10.1155/2020/1250827

Downs, A. (1962). The Law of Peak-Hour Expressway Congestion[J].Traffic, 33(3):347-362. https://doi. org/10.1109/TVC.1965.32997

Gao, K., Han, F., Dong, P., et al. (2019). Connected Vehicle as a Mobile Sensor for Real Time Queue Length at Signalized Intersections[J]. Sensors, 19(9), 2059. https://doi.org/10.3390/s19092059

Gazis, D. C. (1964). Optimum control of a system of oversaturated intersections. Operations Research, 12(6), 807-1051. https://doi.org/10.1287/opre.12.6.815

Jiang, T., Cai, M., Zhang, Y., \& jia, X. (2019). A fast video-based queue length detection approach for selforganizing traffic control. IET Intelligent Transport Systems, 13(4), 670-676. https://doi.org/10.1049/ iet-its.2018.5073

Li, C., \& Fu, Y. (2020). Vehicle Position Correction: A Vehicular Blockchain Networks-Based GPS Error Sharing Framework[J]. IEEE Transactions on Intelligent Transportation Systems, 99, 1-15. https:// doi.org/10.1109/TITS.2019.2961400

Lighthill, M. J., \& Whitham, G. B. (1955). On kinematic waves. II. A theory of traffic flow on long crowded roads, in: Proceedings of the Royal Society of London. Series A. Mathematical and Physical Sciences, :317-345. https://doi.org/10.1098/rspa.1955.0089

Liu, X., Wu, X., Ma, W., \& Hu, H. (2009). Real-time queue length estimation for congested signalized intersections. Transportation Research Part C: Emerging Technologies, 17(4), 412-417. https://doi. org/10.1016/j.trc.2009.02.003

Madhu, K., Sivanandan, R., \& Srinivasan, K. K. (2020). Identification of different vehicle-following manoeuvres for heterogeneous weak-lane disciplined traffic condition from vehicle trajectory data, IOP Conference Series Earth and Environmental Science 491, 012052. https://doi. org/10.1088/1755-1315/491/1/012052

Mehdizadeh, M., \& Shariat-Mohaymany, A. (2020). Who are more likely to break the rule of congestion charging? Evidence from an active scheme with no referendum voting[J]. Transportation Research Part A Policy and Practice, 135. https://doi.org/10.1016/j.tra.2020.03.008

Ortego, J., Andara, R., Navas, L. M., et al. (2020). Impact of the Covid-19 Pandemic on Traffic Congestion in Latin American Cities: An Updated Five-Month Study[J]. Cham: Springer. https://doi. org/10.1007/978-3-030-69136-3_15

Pigou, A. C. (1920). The Economics of Welfare [M] (pp. 70-73). London: MacMillan

Semuel, Y. R., Rompis, M., Cetin, F., \& Habtemichael (2018). Probe vehicle lane identification for queue length estimation at intersections. Journal of Intelligent Transportation Systems, 22(1), 10-25. https://doi.org/10.1080/15472450.2017.1300887

Tan, C., Yao, J., Tang, K., et al. (2021). Cycle-Based Queue Length Estimation for Signalized Intersections Using Sparse Vehicle Trajectory Data[J].IEEE Transactions on Intelligent Transportation Systems, 22 (1):91-106. https://doi.org/10.1109/TITS.2019.2954937. 
Vickrey, W. S. (1969). Congestion theory and transport investment[J]. American Economic Review, 59, 251-261

Wang, C., Sun, Q., Li, Z., \& Zhang, H. (2019). Cognitive competence improvement for autonomous vehicles: a lane change identification model for distant preceding vehicles. IEEE Access, 99, 1-1. https://doi.org/10.1109/ACCESS.2019.2924557

Wang, D., Miwa, T., \& Morikawa, T. (2020a). Big trajectory data mining: a survey of methods, applications, and services, Sensors, 20 (16):4571. https://doi.org/10.3390/s20164571

Wang, Z., Zhu, L., Ran, B., et al. (2020b). Queue profile estimation at a signalized intersection by exploiting the spatiotemporal propagation of shockwaves[J]. Transportation Research Part B: Methodological, 141, 59-71. https://doi.org/10.1016/j.trb.2020b.08.009

Zhang, C., Qin, P., \& Yu, Z. (2020). Research on lane identification based on deep learning. Journal of Computational Methods in Sciences and Engineering, 20(1), 3-11. https://doi.org/10.3233/ JCM-193593

Publisher's Note Springer Nature remains neutral with regard to jurisdictional claims in published maps and institutional affiliations.

\section{Authors and Affiliations}

\section{Wang $\mathrm{Yu}^{1,2} \cdot$ Zhang Dongbo ${ }^{3} \cdot$ Zhang $\mathrm{Yu}^{4}$}

Zhang Dongbo

db_zhang2013@163.com

Wang Yu

sharonawang@163.com

Zhang Yu

y.zhang@giim.ac.cn

1 Key Laboratory for Traffic and Transportation Security of Jiangsu Province, Huai'an, Jiangsu, China

2 Faculty of Transportation Engineering, Huaiyin Institute of Technology, Huai'an, Jiangsu, China

3 School of computer and Information Engineering, Guangdong Polytechnic of Industry and Commerce, Guangzhou, Guangdong, China

4 Institute of Intelligent Manufacturing, Guangdong Academy of Sciences, Guangzhou, Guangdong, China 